\title{
CORRECTIONS
}

\section{Diabetic ketoacidosis in adults}

On 30 October 2015, we replaced the original flow diagram (figure 1) in this Clinical Review (BMJ 2015;351:h5660, doi:10. $1136 /$ bmj.h5660) with a graphic representation of diabetic ketoacidosis (DKA). We thought that this was clearer than the flow diagram. The graphic representation also appeared in the print issue.

Cite this as: BMJ 2015;351:h5866

๑ BMJ Publishing Group Ltd 2015 